\title{
Efectos de una Dieta con Alto Contenido de Grasas sobre Parámetros Alimentarios y Tejido Adiposo Blanco Visceral de Ratas Sprague Dawley
}

\author{
Effects of a High Fat Diet on Dietary Parameters and \\ Visceral White Adipose Tissue of Sprague Dawley Rats
}

Carla Guzmán ${ }^{1,5}$; Katherine Forman ${ }^{2,3}$ \& Raúl Sanchez $^{4,5}$

GUZMÁN, C.; FORMAN, K. \& SANCHEZ, R. Efectos de una dieta con alto contenido de grasas sobre parámetros alimentarios y tejido adiposo blanco visceral de ratas Sprague Dawley. Int. J. Morphol., 38(3):611-615, 2020.

RESUMEN: El incremento en las cifras de obesidad se debe esencialmente a factores de carácter ambiental asociados al consumo de alimentos con alto contenido de grasas saturadas. El objetivo del trabajo fue evaluar el efecto de una dieta alta en grasas sobre parámetros alimentarios y tejido adiposo blanco visceral. Se utilizaron ratas macho Sprague Dawley $(\mathrm{n}=10)$, divididas en dos grupos experimentales, el grupo control recibió dieta convencional (DC) y el grupo experimental una dieta alta en grasas (HFD), durante 10 semanas. Se determinó peso corporal, ingesta alimentaria, conversión alimenticia y características de tejido adiposo. El análisis de datos se realizó utilizando software IBM SPSS versión 21; tras evaluación de la normalidad de los datos, se aplicaron pruebas paramétricas T para muestras independientes y ANOVA de dos vías para medidas repetidas en uno de los factores, con ajuste Bonferroni. Se observó que el promedio de peso fue mayor en los animales alimentados con HFD, sin diferencia estadística respecto a DC, no obstante, existen diferencias significativas en el peso de las ratas alimentadas con HFD en distintos tiempos del protocolo, específicamente semanas 1, 5 y 10 ( $<<0,001)$. La ingesta alimentaria fue mayor en los animales alimentados con DC ( $<<0,005)$, sin embargo el consumo de energía fue mayor en aquellos alimentados con HFD ( $\mathrm{p}=0,016)$, lo que derivó en una mayor conversión alimenticia $(\mathrm{p}<0,005)$. El promedio de diámetro teórico calculado de los adipocitos es estadísticamente mayor en grupo HFD ( $<<0,005)$, lo que se relaciona a la hipertrofia clásica generada tras un período de alimentación con elevado contenido de grasas. Conclusión: El protocolo permite establecer que efectivamente, dado la mayor densidad energética, HFD induce hipertrofia de los adipocitos, proceso característico de la obesidad.

PALABRAS CLAVE: Tejido adiposo; Obesidad; Dieta alta en grasas.

\section{INTRODUCCIÓN}

En la actualidad la obesidad es un grave problema de salud a nivel mundial (Tsai et al., 2011), ya que ha alcanzado proporciones epidémicas en las últimas dos décadas, convirtiéndose en una patología de elevada morbilidad y mortalidad (Weigl \& Holzapfel, 2016). El incremento acelerado en las tasas de malnutrición por exceso, se debe esencialmente a factores de carácter ambiental asociados al consumo de alimentos con alto contenido de grasas saturadas, producto de la mayor elaboración e industrialización de los alimentos (Atalah, 2012). Esto genera presencia de lipotoxicidad, que se define como la acumulación ectópica de lípidos en órganos periféricos no adiposos, siendo un factor determinante del estrés metabólico (Valle et al., 2019) y de una grave desregulación a nivel orgánico caracterizado por la producción anormal de adipocitocinas, presencia de estrés oxidativo y respuesta proinflamatoria crónica (Kintscher et al., 2008), contribuyendo en el desarrollo de eventos mórbidos, tales como diabetes mellitus tipo II, hipertensión arterial y dislipidemia. El objetivo de esta investigación fue evaluar el efecto de una dieta alta en grasas sobre parámetros alimentarios y tejido adiposo blanco visceral de ratas Sprague Dawley, respecto a aquellas alimentadas con una dieta convencional. Las variables estudiadas fueron ganancia de peso acumulada, ingesta alimentaria, ingesta energética, conversión alimenticia y características del tejido adiposo blanco visceral.

\footnotetext{
${ }^{1}$ Carrera de Nutrición y Dietética, Facultad Ciencias para el Cuidado de la Salud, Universidad San Sebastián, Concepción, Chile.

${ }^{2}$ Centro de Microscopía Avanzada, CMA Bio-Bio, Facultad de Ciencias Biológicas, Universidad de Concepción, Concepción, Chile.

${ }^{3}$ Departamento de Nutrición y Dietética, Facultad de Farmacia, Universidad de Concepción, Concepción, Chile.

${ }^{4}$ Departamento de Ciencias Preclínicas, Facultad de Medicina, Universidad de La Frontera, Temuco, Chile.

${ }^{5}$ Programa de Doctorado en Ciencias Médicas, Universidad de La Frontera, Temuco, Chile.
} 


\section{MATERIAL Y MÉTODO}

Animales: Se utilizaron 10 ratas macho Sprague Dawley de 6 - 7 semanas de edad, obtenidas en el Centro Regional de Estudios Avanzados para la Vida, ubicado en Universidad de Concepción. Se alojaron en jaulas metálicas con ambiente enriquecido, bajo condiciones controladas de temperatura $25+1^{\circ} \mathrm{C}$ y humedad $60 \%$, con ciclo de luz/oscuridad de 12 horas. La investigación se llevó a cabo bajo los lineamientos de la Guía para el cuidado y uso de animales de laboratorio (National Research Council, 2011) y fue aprobada por el Comité Ético Científico de la Universidad de La Frontera (Folio No 113_17).

Dietas: Tras una semana de aclimatación con acceso a dieta convencional y agua ad libitum, los animales fueron divididos aleatoriamente en 2 grupos. Se utilizó en cada grupo experimental un $n=5$, considerando los Principios Bioéticos Russel y Burch: reemplazo, reducción y refinamiento (Tannenbaum \& Bennett, 2015). El grupo control recibió dieta convencional con densidad energética de 3 $\mathrm{kcal} / \mathrm{g}$, elaborada por Labdiet (USA). El grupo experimental recibió dieta alta en grasas con densidad energética de $5 \mathrm{kcal} / \mathrm{g}$, elaborada por PragSolutions Serviços e Com. Ltda. Pellets (São Paulo, Brasil) la que fue almacenada a $-20^{\circ} \mathrm{C}$ para su conservación durante todo el estudio. El protocolo descrito se llevó a cabo durante 10 semanas (Gregorio et al. 2013; Fernandes-Lima et al., 2016). La distribución de macronutrientes de ambas dietas se describe en la Tabla I.

Tabla I. Distribución de macronutrientes de las dietas utilizadas.

\begin{tabular}{lcc}
\hline \multicolumn{1}{c}{ Macronutrientes } & DC & HFD \\
\hline Proteínas & $14 \%$ & $19 \%$ \\
Carbohidratos & $76 \%$ & $32 \%$ \\
Lípidos & $10 \%$ & $49 \%$ \\
Densidad energética & $3 \mathrm{kcal} / \mathrm{g}$ & $5 \mathrm{kcal} / \mathrm{g}$ \\
\hline DC: Dieta convencional; HFD: Dieta alta en grasas.
\end{tabular}

Biometría y variables alimentarias: Se determinó peso corporal (g) de cada animal una vez a la semana durante todo el protocolo experimental, mientras que la ingesta alimentaria sólida (g) y líquida (ml) se registró en forma diaria, representando el valor medio por jaula ponderada por el número de animales. La ingesta energética se cuantifica a partir de la ingesta sólida de cada animal, la ganancia acumulada sumando el aumento de peso corporal de cada semana y la conversión alimenticia se expresa como la relación obtenida entre la cantidad de alimento consumido y la ganancia de peso diaria por animal (Olguin et al., 2015).
Eutanasia: Al finalizar la intervención y tras un ayuno de 8 horas se llevó a cabo la eutanasia mediante saturación de gas con una campana de Isoflurano, confirmando visualmente el estado de inconciencia. Se ubicó al animal en cúbito dorsal para la exanguinación por punción cardíaca. La sangre, dispuesta en tubos con heparina de litio, se centrifugó (3000 rpm durante 15 minutos) a $4{ }^{\circ} \mathrm{C}$, almacenando el plasma a $-80{ }^{\circ} \mathrm{C}$ para análisis posteriores.

Estudio morfológico: Inmediatamente tras la eutanasia, se llevó a cabo la extracción de tejido adiposo blanco visceral, tomando 3 cortes de 3-4 $\mathrm{mm}^{2}$ por animal los que fueron fijados por inmersión en solución Bouin (ácido pícrico, formalina y ácido acético), incluidos en parafina, montados sobre portaobjetos de vidrio recubierto de poli-L-lisina y procesados para obtener cortes seriados de $7 \mathrm{~mm}$ en micrótomo 98.

Análisis estadístico: El análisis de datos se realizó utilizando software IBM SPSS versión 21. La normalidad de la distribución de los datos se determinó a través de la prueba Shapiro Wilk. La ganancia de peso, ingesta alimentaria, consumo energético, conversión alimenticia y características de adipocitos entre ambos grupos se analizó mediante prueba $\mathrm{T}$ para muestras independientes, mientras que la ganancia acumulada del grupo alimentado con dieta alta en grasas se analizó mediante prueba ANOVA de dos vías para medidas repetidas en uno de los factores, con ajuste Bonferroni. Los datos fueron representados por media + SEM, se consideró significancia estadística con $\mathrm{p}<0,05$.

\section{RESULTADOS Y DISCUSIÓN}

Efecto de la dieta alta en grasas sobre peso corporal, ingesta alimentaria, ingesta energética y conversión alimenticia. La dieta alta en grasas se asocia con aumento de peso corporal e inflamación crónica producto de la infiltración de macrófagos en el tejido adiposo (Kim et al., 2012; Navarrete et al., 2018). En este estudio se analiza el aumento de peso registrado en los grupos DC y HFD, obteniéndose un promedio de $441,96 \pm 11,3 \mathrm{~g}$ y $461,94 \pm 18,0 \mathrm{~g}$ respectivamente, lo que representa una ganancia de peso acumulado mayor en aquellos animales alimentados con HFD, respecto a DC. Dado que existe normalidad en los datos, se aplica prueba paramétrica $\mathrm{T}$ student para muestras independientes, sin diferencias entre ambos grupos (Tabla II), no obstante existen diferencias significativas en la ganancia de peso entre distintos tiempos del protocolo en el grupo HFD, específicamente en semanas 1,5 y 10 , analizado a través de ANOVA de medidas repetidas y prueba posthoc Bonferroni ( $p<0,001)$ (Fig. 1). 
La ingesta alimentaria en ambos grupos promedió $27,66 \pm 0,3 \mathrm{~g}$ y $19,73 \pm 0,8 \mathrm{~g}$ en aquellos alimentados con DC y HFD respectivamente, con diferencia significativa tras aplicación de T student para muestras independientes $(\mathrm{p}<0,005)$ (Fig. 2), siendo mayor en aquellos animales alimentados con DC, quienes tienen menor peso corporal, lo que se explica dado que al consumir alimentos de menor densidad energética, los animales requieren el consumo de un gramaje mayor para lograr el mismo nivel de saciedad que con HFD. Respecto al consumo de energía, fue mayor en aquellos alimentados con HFD ( $\mathrm{p}=0,016)$ (Fig. 3), lo que derivó en una mayor conversión alimenticia $(p<0,005)$ (Fig. 4), que se asocia a la eficiencia de HFD para inducir aumento de peso corporal.

Tabla II. Efecto de HFD sobre peso corporal y variables alimentarias.

\begin{tabular}{lcc}
\hline \multicolumn{1}{c}{ Protocolo } & DC & HFD \\
\hline Peso promedio $(\mathrm{g})$ & $441,96 \pm 11,3$ & $461,94 \pm 18,0$ \\
Ganancia de peso $(\mathrm{g})$ & $171,80 \pm 10,9$ & $231,8 \pm 17,4$ \\
Ingesta sólida $(\mathrm{g})$ & $27,66+0,3$ & $19,73+0,8 *$ \\
Ingesta líquida $(\mathrm{ml})$ & $31,08 \pm 0,3$ & $22,9 \pm 1,2 *$ \\
Ingesta energética $(\mathrm{kcal})$ & $82,99 \pm 1,03$ & $98,68 \pm 4,03 *$ \\
Conversión alimenticia & $0,69 \pm 0,03$ & $1,31 \pm 0,05 *$ \\
\hline
\end{tabular}

DC: Dieta convencional; HFD: Dieta alta en grasas. *Diferente del grupo DC ( $<<0,005$, Prueba T para igualdad de medias).

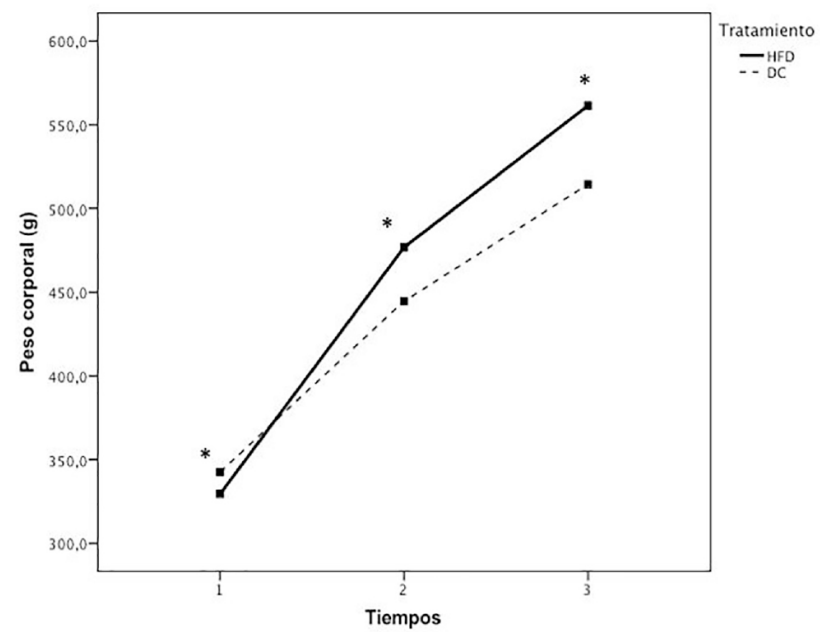

Fig. 1. Evolución peso corporal. DC: Dieta convencional; HFD: Dieta alta en grasas. *Diferente del grupo DC en 3 tiempos ( $p<0,001$, Prueba ANOVA para medidas repetidas)

Efecto de dieta alta en grasas sobre el número y tamaño de adipocitos. El tejido adiposo se expande mediante mecanismos de hipertrofia, aumentando el tamaño de los adipocitos, lo que potencia la expresión y secreción de citocinas proinflamatorias que conducirán a la fosforilación del sustrato de serina 1 del receptor de insulina (IRS-1) a través de la activación del factor nuclear kappa b (NFkbb), provocando insulino resistencia (Giordano et al. 2013). Se cuantificó la cantidad de adipocitos por área de los grupos

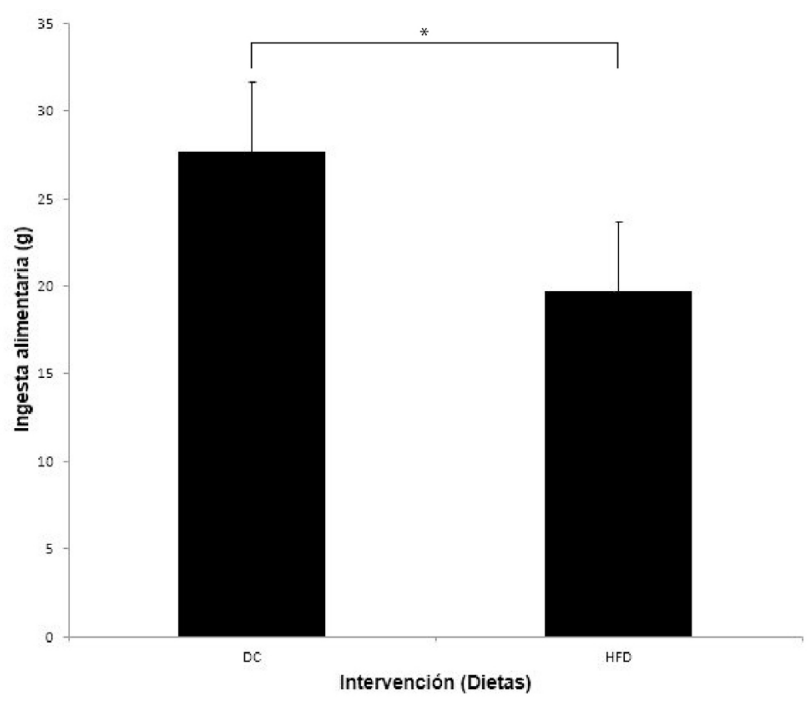

Fig. 2. Promedio ingesta alimentaria . DC: Dieta convencional; HFD: Dieta alta en grasas. *Diferente del grupo DC ( $p<0,005$ Prueba $\mathrm{T}$ para igualdad de medias).

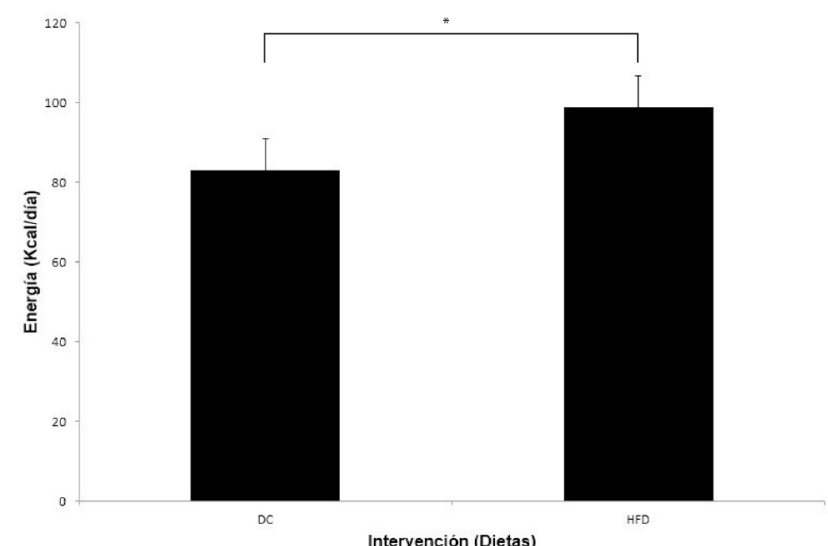

Fig. 3. Promedio energía consumida . DC: Dieta convencional; HFD: Dieta alta en grasas. *Diferente del grupo DC (p 0,016 Prueba $\mathrm{T}$ para igualdad de medias).

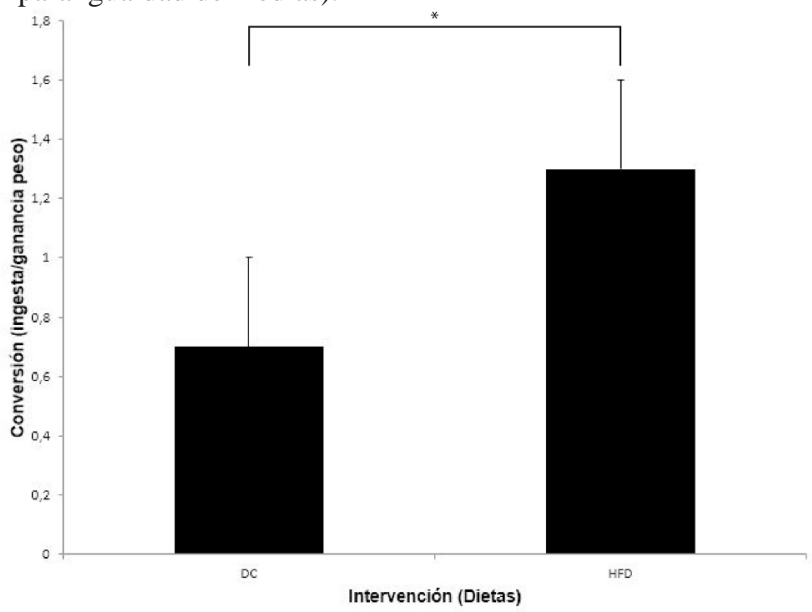

Fig. 4. Promedio conversión alimenticia. DC: Dieta convencional; HFD: Dieta alta en grasas. *Diferente del grupo DC en cada semana ( $p<0,005$ Prueba $T$ para igualdad de medias). 
DC y HFD, obteniéndose en promedio 40,5 $\pm 4,26$ y $33,0 \pm$ 5,97 respectivamente, sin diferencias entre ambos, no obstante el promedio de diámetro teórico calculado es estadísticamente mayor en aquellos animales alimentados con HFD (Tabla III; Fig. 5), hallazgo relacionado con la hipertrofia clásica observada tras un período regular de alimentación con elevado contenido de grasas (Jo et al. 2009).
Tabla III. Efecto de HFD sobre características de tejido adiposo blanco.

\begin{tabular}{lcc}
\hline \multicolumn{1}{c}{ Protocolo } & DC & HFD \\
\hline Número de células & $40,5+4,26$ & $33,0+5,07$ \\
Diámetro teórico (um) & $65,9+1,85$ & $81,6+5,60 *$
\end{tabular}

DC: Dieta convencional; HFD: Dieta alta en grasas. *Diferente del grupo $\mathrm{DC}(\mathrm{p}<0,005$, Prueba $\mathrm{T}$ para igualdad de medias).
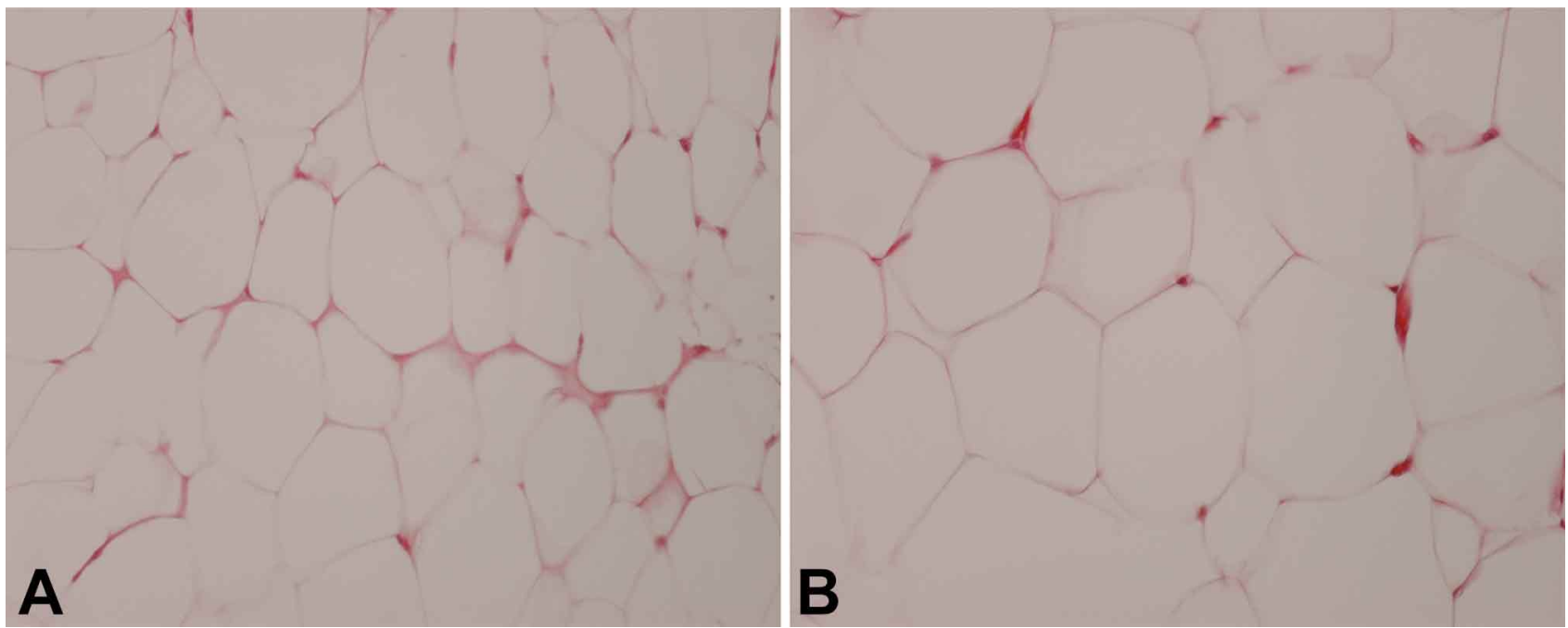

Fig. 5. Tejido adiposo blanco visceral. A) Corte transversal de tejido adiposo blanco visceral de ratas sometidas a alimentación convencional durante 10 semanas. 20X B) Corte transversal de tejido adiposo blanco visceral de ratas sometidas a alimentación alta en grasas durante 10 semanas. $20 \mathrm{X}$

\section{CONCLUSIÓN}

El aumento del compartimento graso, particularmente visceral, se debe esencialmente a factores de carácter ambiental, dado por la elevada ingesta de alimentos procesados cuyo componente principal son las grasas saturadas. En este estudio se refuerza la evidencia que efectivamente HFD, producto de su mayor densidad energética y conversión alimenticia, provoca hipertrofia adipocitaria, proceso característico de la obesidad y por tanto factor etiológico relevante de desórdenes metabólicos propios de la malnutrición por exceso.

GUZMÁN, C.; FORMAN, K. \& SANCHEZ, R. Effects of a high fat diet on dietary parameters and visceral white adipose tissue of Sprague Dawley rats. Int. J. Morphol., 34(3):611-615, 2020.

SUMMARY: The continued increase in obesity statistics is the result of environmental factors associated with the consumption of foods high in saturated fat. The objective of the work was to evaluate the effect of a high fat diet on food parameters and visceral white adipose tissue. in Male Sprague Dawley rats $(\mathrm{n}=10)$ were used, divided into two experimental groups, the control group received conventional diet (DC) and the experimental group a high fat diet (HFD), for 10 weeks. Body weight, food intake, food conversion and adipose tissue characteristics were determined. Data analysis was performed using IBM SPSS version 21 software; after evaluating the normality of the data, parametric $\mathrm{T}$ tests were applied for independent samples and two-way ANOVA for repeated measurements in one of the factors, with Bonferroni adjustment. It was observed that the average weight was higher in animals fed with HFD, without statistical difference with respect to DC, however, there were significant differences in the weight of rats fed with HFD at different times of the protocol, specifically weeks 1.5 and 10 ( $\mathrm{p}<0.001)$. Food intake was higher in animals fed DC ( $p<0.005$ ), however the energy consumption was higher in those fed with HFD ( $\mathrm{p}=0.016)$, which resulted in a higher feed conversion $(p<0.005)$. The average theoretical diameter calculated for adipocytes is statistically higher in the HFD group ( $p<0.005$ ), which is related to the classical hypertrophy generated after a period of feeding with high fat content. In conclusion, the protocol allows us to establish that, given the higher energy density, HFD induces adipocyte hypertrophy, a characteristic in the obesity process.

\section{KEY WORDS Adipose tissue; Obesity; Diet.}




\section{REFERENCIAS BIBLIOGRÁFICAS}

Atalah, S. E. Epidemiología de la obesidad en Chile. Rev. Med. Clin. Las Condes, 23(2):117-23, 2012.

Fernandes-Lima, F.; Monte, T. L. R. G.; de Morais Nascimento, F. A. \& Gregório, B. M. Short exposure to a high-sucrose diet and the first 'hit' of nonalcoholic fatty liver disease in mice. Cell Tissues Organs, 201(6):464-72, 2016.

Giordano, A.; Murano, I.; Mondini, E.; Perugini, J.; Smorlesi, A.; Severi, I.; Barazzoni, R.; Scherer, P. R. \& Cinti, S. Obese adipocytes show ultrastructural features of stressed cells and die of pyroptosis. J. Lipid Res., 54(9):2423-36, 2013.

Gregorio, B. M.; Souza-Mello, V.; Mandarim-de-Lacerda, C. A. \& Aguila, M. B. Maternal high-fat diet is associated with altered pancreatic remodelling in mice offspring. Eur. J. Nutr., 52(2):759-69, 2013.

Jo, J.; Gavrilova, O.; Pack, S.; Jou, W.; Mullen, S.; Sumner, A. E.; Cushman, S. W. \& Periwal, V. Hypertrophy and/or hyperplasia: dynamics of adipose tissue growth. PLoS Comput. Biol., 5(3):e1000324, 2009.

Kim, K., Gu, W., Lee, I., Joh, E., \& Kim, D. High fat diet-induced gut microbiota exacerbates inflammation and obesity in mice via the TLR4 signaling pathway. PLoS One, 7(10):e47713, 2012.

Kintscher, U.; Hartge, M.; Hess, K.; Foryst-Ludwig, A.; Clemenz, M.; Wabitsch, M.; Fischer-Posovszky, P.; Barth, T. F. E.; Dragun, D.; Skurk, T.; et al. T-lymphocyte infiltration in visceral adipose tissue: a primary event in adipose tissue inflammation and the development of obesitymediated insulin resistance. Arterioscler. Thromb. Vasc. Biol., 28(7):1304-10, 2008.

National Research Council. Committee for the Update of the Guide for the Care and Use of Laboratory Animals. Guide for the Care and Use of Laboratory Animals. $8^{\text {th }}$ ed. Washington (WD), National Research Council, 2011.

Navarrete, J.; Vásquez, B.; Vasconcellos, A.; del Sol, M.; Olave, E. \& Sandoval, C. Effects of high-fat diets on biochemical profiles and morpho- quantitative characteristics of C57BL/6 mice adrenal glands. Int. J. Morphol., 36(2):722-9, 2018.

Olguin, B. M. C.; Posadas, R. M. D.; Revelant, Z. G. C.; Labourdette, P. V.; Marinozzi, T. D. O.; Venezia, N. M. R. \& Zingale, V. M. I. Effects of a high fructose and sucrose intake on metabolic parameters in obese diabetic rats. Rev. Chil. Nutr., 42(2):151-6, 2015.

Saout, C.; Quere, C.; Donval, A.; Paulet, Y. M. \& Samain, J. F. An experimental study of the combined effects of temperature and photoperiod on reproductive physiology of pecten maximus from the bay of brest (France). Aquaculture, 172:301-14, 1999.

Tannenbaum, J. \& Bennett, B. T. Russell and Burch's 3Rs then and now: the need for clarity in definition and purpose. J. Am. Assoc. Lab. Anim. Sci., 54(2):120-32, 2015.

Tsai, A. G.; Williamson, D. F. \& Glick, H. A. Direct medical cost of overweight and obesity in the USA: a quantitative systematic review. Obes. Rev., 12(1):50-61, 2011.

Valle, A. D.; Vertongen, P.; Spruyt, D.; Lechanteur, J.; Suain, V.; Gaspard, N.; Brion, J. P.; Gangji, V. \& Rasschaert, J. Induction of stearoyl-CoA 9-desaturase 1 protects human mesenchymal stromal cells against palmitic acid-induced lipotoxicity and inflammation. Front. Endocrinol. (Lausanne), 10:726, 2019.

Weigl, J. \& Holzapfel, C. Body Mass Index makes headlines - When is mortality rate lowest? Dtsch. Med. Wochenschr., 141(14):1040-1, 2016.

\author{
Dirección para correspondencia: \\ Carla Guzmán \\ Lientur 1457 \\ Concepción 4080871 \\ CHILE
}

Email: carla.guzman@uss.cl

Recibido : 15-11-2019

Aceptado: 27-12-2019 